\title{
Robust transcriptional signatures for low- input RNA samples based on relative expression orderings
}

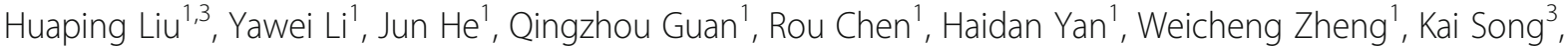 \\ Hao Cai ${ }^{1}$, You Guo ${ }^{1}$, Xianlong Wang ${ }^{1 *}$ and Zheng Guo ${ }^{1,2,3,4^{*}}$
}

\begin{abstract}
Background: It is often difficult to obtain sufficient quantity of RNA molecules for gene expression profiling under many practical situations. Amplification from low-input samples may induce artificial signals.

Results: We compared the expression measurements of low-input mRNA samples, from 25 pg to 1000 pg mRNA, which were amplified and profiled by Smart-seq, DP-seq and CEL-seq techniques using the Illumina HiSeq 2000 platform, with those of the paired high-input (50 ng) mRNA samples. Even with 1000 pg mRNA input, we found that thousands of genes had at least 2 folds-change of expression levels in the low-input samples compared with the corresponding paired high-input samples. Consequently, a transcriptional signature based on quantitative expression values and determined from high-input RNA samples cannot be applied to low-input samples, and vice versa. In contrast, the within-sample relative expression orderings (REOs) of approximately $90 \%$ of all the gene pairs in the high-input samples were maintained in the paired low-input samples with 1000 pg input mRNA molecules. Similar results were observed in the low-input total RNA samples amplified and profiled by the Whole-Genome DASL technique using the Illumina HumanRef-8 v3.0 platform. As a proof of principle, we developed REOs-based signatures from high-input RNA samples for discriminating cancer tissues and showed that they can be robustly applied to low-input RNA samples.
\end{abstract}

Conclusions: REOs-based signatures determined from the high-input RNA samples can be robustly applied to samples profiled with the low-input RNA samples, as low as the $1000 \mathrm{pg}$ and $250 \mathrm{pg}$ input samples but no longer stable in samples with less than $250 \mathrm{pg}$ RNA input to a certain degree.

Keywords: Low-input RNA samples - amplification artificial signals - relative expression orderings - transcriptional signatures

\section{Background}

Gene expression profiling based on microarray and RNA sequencing techniques allows us to comprehensively characterize RNA transcripts present in a biological sample. However, it is often difficult to obtain sufficient quantity of RNA molecules for gene expression profiling under many practical situations. For example, minimally invasive tissue biopsy techniques, such as fine needle aspiration cytology, core needle biopsy and gastrointestinal endoscopy,

\footnotetext{
* Correspondence: wang.xianlong@139.com; guoz@ems.hrbmu.edu.cn 'Department of Bioinformatics, Key Laboratory of Ministry of Education for Gastrointestinal Cancer, School of Basic Medical Sciences, Fujian Medical University, Fuzhou 350122, China

Full list of author information is available at the end of the article
}

are widely used clinically but minimum samples are extracted [1-3]. For another example, in formalin-fixed paraffin-embedded tissue samples with abundant clinical information, the amount of RNA is often limited due to partial RNA degradation $[4,5]$. In the studies of rare cell population, single cell $[6,7]$ or the samples taken with the laser capture microdissection [8] technique, the amount of RNA molecules is also extremely low.

It is critical to overcome this challenge to leverage the power of low-input sampling techniques for biomedical applications. For this type of samples, multiple rounds of pre-amplification are necessary prior to the measurements of gene expression levels. Thus, a number of low- 
input RNA amplification techniques prior sequencing have been developed using PCR or in vitro transcription (IVT) to synthesize enough cDNA or cRNA, such as Smart-seq (switching mechanism at 5 '-end of the RNA transcript) [9], DP-seq (primer-based RNA-sequencing strategy) [10] and CEL-seq (cell expression by linear amplification and sequencing) [11]. However, current low-input amplification techniques usually bring a large bias due to the inherent defects in the amplification principles [12]. For example, CEL-seq incorporating IVT can result in 3' biases due to two rounds of reverse transcription before the linear amplification $[11,13]$. Smart-Seq, using PCR to synthesize cDNA, is a nonlinear amplification process, and its efficiency is sequencedependent $[9,13]$; a long transcript may be truncated due to inefficient cDNA synthesis during the amplification process $[14,15]$. It has been reported that the amplification bias always exists in lowly expressed genes and genes with abundant CG and long length [16-18]. As a result, it is uncertain whether the expression values measured after the amplification can represent the real gene expression levels or not.

Several studies attempted to prove that gene expression profiling can be performed on low-input RNA samples like high-input RNA samples by showing that the gene expression profiles of low-input RNA samples are significantly correlated with those of the matched highinput RNA samples [19-21]. However, a high correlation between two measurements does not guarantee that the two measurements are congruent, which brings uncertainty to the application of most current disease signatures based on risk scores which are calculated using the measurement values of the signature genes. Therefore, for a transcriptional signature based on the quantitative expression levels, the risk score thresholds determined from high-input RNA samples may be not applicable to low-input RNA samples, and vice versa. It has been reported that quantitative transcriptional signatures lack robustness for clinical applications due to measurement batch effects [22], variations of the tumor epithelial cell proportions in tissues sampled from different sites of a tumor $[23,24]$ and partial RNA degradation during sample preparation $[25,26]$. Another type of disease signature is based on the within-sample relative expression orderings (REOs) of gene pairs [27, 28], which have been identified for predicting the prognosis of colorectal cancer [29], non-small cell lung cancer [30], ER+ breast cancer [31] and other cancers [32, 33]. These REOsbased signatures are robust against various measurement biases introduced by experimental batch effects and platform differences [34], partial RNA degradation [26] and uncertain sampling sites within the same cancer tissue [24]. Thus, we hypothesized that the REOs of gene pairs within individual samples, especially those with large rank differences, might also be robust against the biases introduced by the RNA amplification procedures.

Through comparing gene expression profiles between the samples with low-input mRNA, ranging from $25 \mathrm{pg}$ to 1000 pg mRNA profiled by the Illumina HiSeq 2000 platform, and their paired high-input 50 ng mRNA samples, we found that there were thousands of genes with at least 2 folds-change (FC) in their expression values even when the input mRNA was $1000 \mathrm{pg}$. We evaluated the proportions of REOs of gene pairs in the high-input RNA samples maintained in the low-input RNA samples, and found that the proportions were approximately $90 \%$ even when the input mRNA was as low as $1000 \mathrm{pg}$ and the input total RNA samples was as low as $250 \mathrm{pg}$, which suggests that REOs measured in the low-input samples were robust against amplification. Similar results were also found in the low input total RNA ranging from $10 \mathrm{pg}$ to $1000 \mathrm{pg}$ profiled by the Illumina HumanRef- 8 v3.0 platform compared with the $100 \mathrm{ng}$ input total RNA samples. As a case study to demonstrate the robustness of REOs-based signatures, we developed REOs-based signatures from highinput RNA samples for discriminating cancer tissues and showed that they can be robustly applied to low-input RNA samples.

\section{Results}

\section{Large amplification bias of low-input RNA samples}

Based on two datasets (GSE50856 and GSE17565, see Fig. 1) measured by Illumina HiSeq 2000 and Illumina HumanRef-8 v3.0 platforms, respectively, we evaluated the amplification fidelity of low-input RNA samples amplified by several techniques through comparison with the corresponding high-input RNA samples using the $\mathrm{FC}$ values.

In the SFM-Smart group of dataset GSE50856, there were respectively $60.56,64.00,65.76$ and $66.95 \%$ of genes with a FC value larger than or equal to 2 in the expression values between $1000 \mathrm{pg}, 100 \mathrm{pg}, 50 \mathrm{pg}$ and $25 \mathrm{pg}$ mRNA samples compared with the paired high-input samples. As the amount of RNA in the diluted low input samples decreased, the percentage of genes with at least 2 FC increased. Similar results were also observed in the other five groups of dataset GSE50856 (Fig. 2). In the SFM-smart data, the coefficient of variation (CV) of FCs increased from 0.18 to 0.33 as the quantity of the input RNA decreased from $1000 \mathrm{pg}$ to $25 \mathrm{pg}$ (Additional file 1: Figure 1Sa). Similar results were observed in the data for SFM-DP, SFM-CEL, AA100-Smart, AA100-DP, AA100CEL, Raji and MCF-7 (Additional file 1: Figure 1Sa, b and c). Thus, a large amplification bias exists for the three amplification techniques even the amplification begins from 1000 pg mRNA input.

For the Raji group of dataset GSE17565, there were $12.02,23.79,41.73,57.84 \%$ of genes with a FC value 

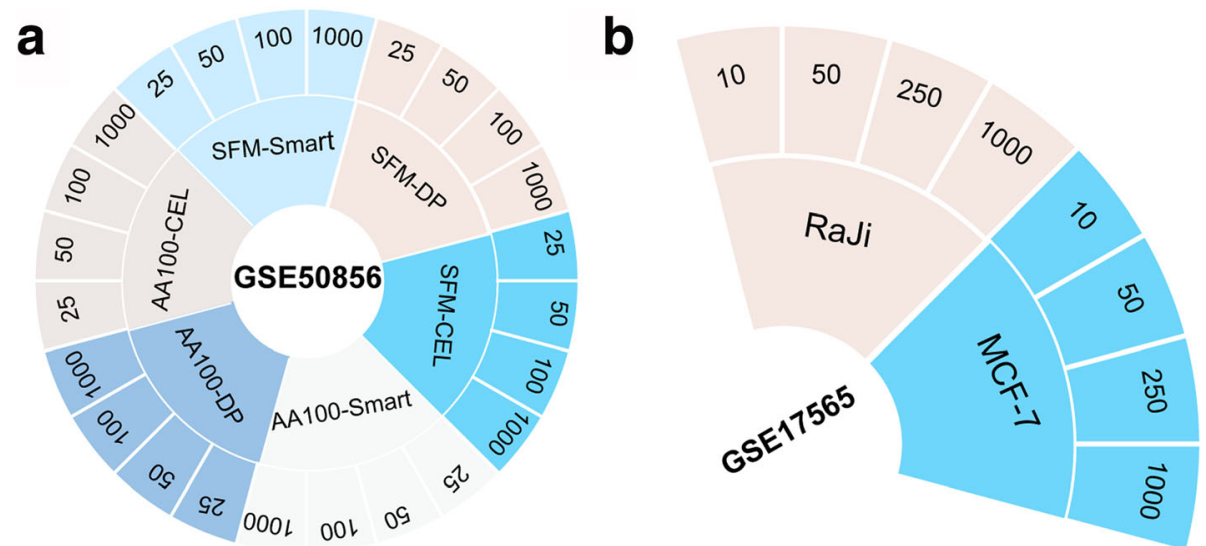

Fig. 1 Datasets in this study. a The GSE50856 dataset was divided into 6 groups: SFM-Smart, SFM-DP, SFM-CEL, AA100-Smart, AA100-DP and AA100-CEL. Each group had four low input mRNA levels, 1000 pg, 100 pg, 50 pg and 25 pg, and each level had two technical replicates. b The GSE17565 dataset was divided into 2 groups: Raji and MCF-7. Each group had four low input total RNA levels, 1000 pg, 250 pg, 50 pg and 10 pg, and each level had two technical replicates

larger than or equal to 2 in the expression values, respectively, in the $1000 \mathrm{pg}, 250 \mathrm{pg}, 50 \mathrm{pg}, 10 \mathrm{pg}$ samples compared with the paired high-input samples. Similar results were also observed for the MCF-7 group (Fig. 2). Obviously, the amplification procedure has a profound negative impact on the measurements of gene expression levels of the low-input samples.

\section{Robustness of REOs against amplification bias}

Using the same datasets, we evaluated the consistency scores between the low-input samples and the highinput samples, i.e. the proportions of the REOs of the gene pairs in the high-input RNA samples maintained in the low-input samples. All genes from the gene expression profiles were involved in the REO gene pairs.

In the SFM-Smart dataset GSE50856, 88.53 and $88.63 \%$ of the stable REOs in the high-input mRNA samples were respectively kept in the two $1000 \mathrm{pg}$ input mRNA technical replicates. Obviously, the REOs of gene pairs with small rank differences (i.e., close expression levels) tend to be sensitive to random measurement variations [34]. After excluding $10 \%$ of pairs with the smallest rank differences, the percentages increased to 91.36 and $91.46 \%$ in the two 1000 pg input mRNA technical replicates, respectively. The percentage of the stable REOs in the high-input samples that were kept in the low input technical replicates, termed the consistency scores for short, decreased gradually when the input mRNA decreased. The consistency scores for the two $100 \mathrm{pg}$ input technical replicates decreased to 85.66 and $85.36 \%$, respectively, and increased to 88.24 and $87.92 \%$ after excluding the bottom $10 \%$ of the gene pairs in the high-input mRNA samples. For the two 50 pg input technical replicates, the consistency scores were 83.84 and $83.29 \%$, respectively, and increased to 86.27 and $85.67 \%$, respectively, after excluding the bottom $10 \%$ of the stable gene pairs. For the two 25 pg input technical replicates, the consistency scores were 82.11 and $81.99 \%$, respectively, and increased to 84.39 and $84.26 \%$ after excluding the bottom $10 \%$ of the gene pairs (Fig. 3a). Similar results were also found in the SFM-DP (Fig. 3b), SFM-CEL (Fig. 3c), AA100-Smart (Additional file 1: Figure S2a), AA100-DP (Additional file 1: Figure S2b) and AA100CEL groups (Additional file 1: Figure S2c). As shown in

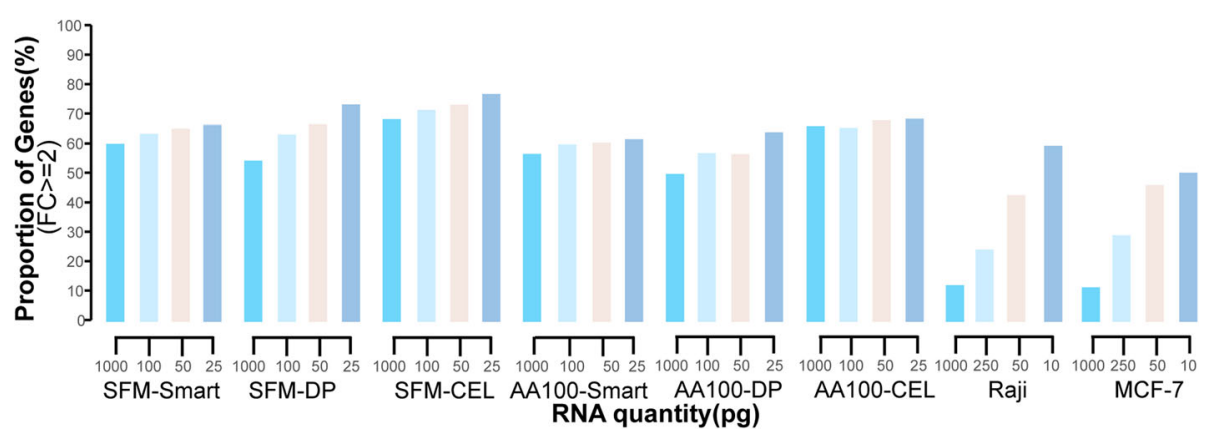

Fig. 2 Amplification bias. Proportion of genes with at least 2 folds-change of expression values 

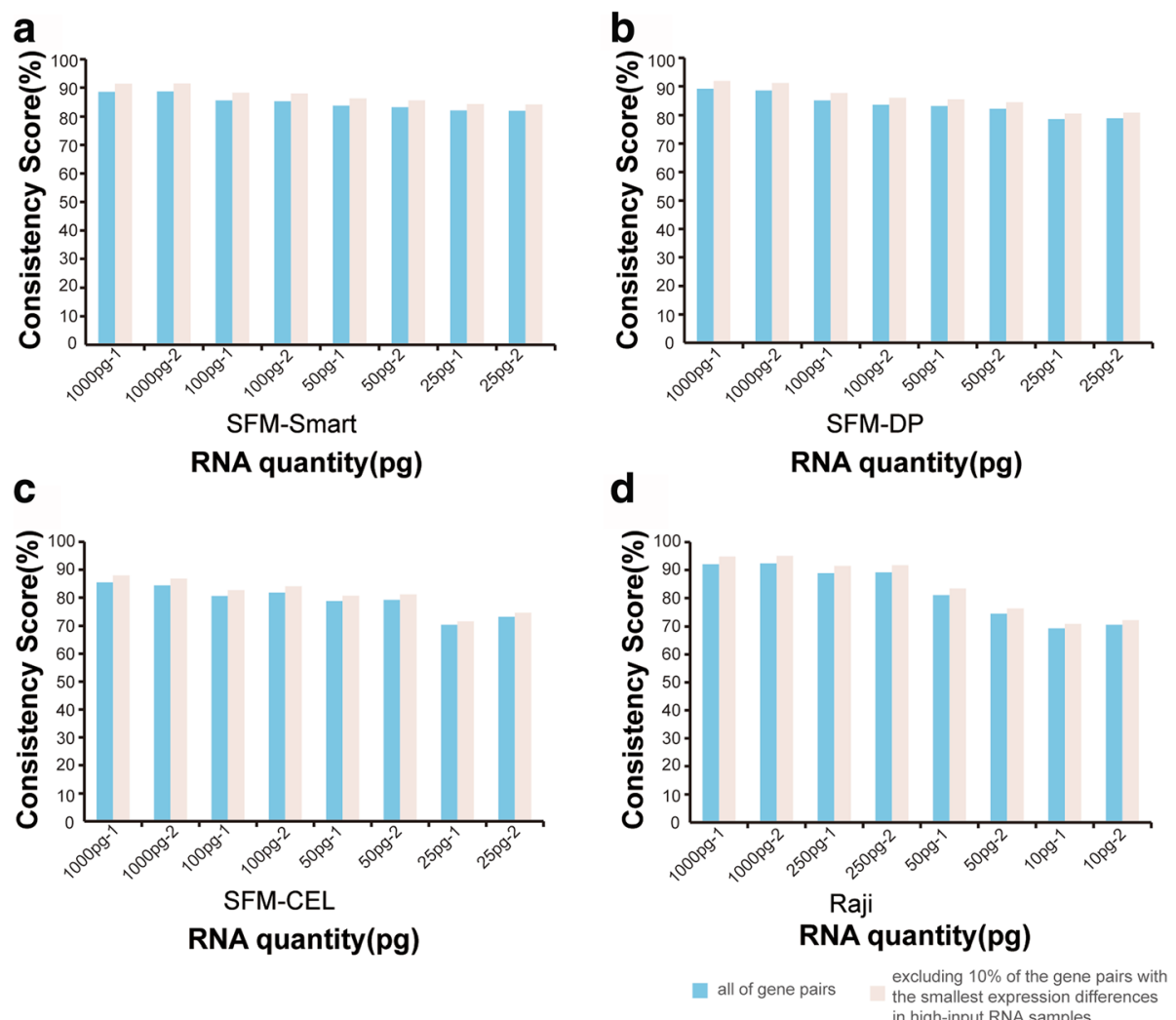

Fig. 3 Maintenance of REOs after excluding 0\% to 10\% gene pairs (a) The consistency scores between high-input RNA samples and low-input RNA samples of all gene pairs (blue) and after excluding 10\% of the pairs with the smallest expression differences in the paired high-input RNA samples (pink) in the group of SFM-Smart (b) (c) (d) Similar as the Fig. a

the (Additional file 1: Figure S3, Figure S4), the percentage of the stable REOs in the high-input samples that were kept in each of the low input technical replicates increased when more gene pairs with small rank differences in the high-input samples were excluded.

For all the 164,238,402 gene pairs which had the same REOs among two technical replicates of the high-input samples in the Raji dataset GSE17565 measured by the Illumina HumanRef-8 v3.0 platform, 92.09 and $92.43 \%$ were respectively kept in the two technical replicates with 1000 pg input total RNA. The consistency scores increased to 94.83 and $95.08 \%$, respectively, after excluding the bottom $10 \%$ gene pairs with the smallest rank differences. For the two $250 \mathrm{pg}$ input technical replicates, the consistency scores for all the stable gene pairs were 88.87 and $89.20 \%$, respectively, and increased to 91.49 and $91.76 \%$ after excluding the bottom $10 \%$ of the gene pairs. For the two $50 \mathrm{pg}$ input technical replicates, the consistency scores were 81.06 and $74.52 \%$, respectively, and increased to 83.39 and $76.42 \%$ after excluding the bottom $10 \%$ of the gene pairs. For the two $10 \mathrm{pg}$ input technical replicates, the consistency scores were 69.32 and $70.58 \%$, respectively, and increased to 70.87 and $72.23 \%$ after excluding the bottom $10 \%$ of the gene pairs (Fig. 3d). Similar results were also found in the MCF-7 group (Additional file 1: Figure S4d).

Taken together, the above results showed that the REOs of gene pairs were robust against the amplification bias for the $1000 \mathrm{pg}$ and $250 \mathrm{pg}$ input samples but no longer stable in samples with less than 250 pg RNA input to a certain degree.

\section{Performance of REOs-based signatures in low-input RNA samples}

As a proof of principle that REOs-based signature identified from high-input RNA tissue samples are robust in low-input RNA samples, we collected 69 high-input RNA samples of lymphoma tissues from the GSE55267 dataset and 54 high-input RNA samples of breast cancer tissues from the GSE29431 dataset to search a REOsbased signature for discriminating the two types of tissues (Table 1). We obtained 106,213 highly stable gene pairs that have the same REOs in all lymphoma tissue samples and breast cancer tissue samples, respectively, but the REO patterns were reversal between the two tissue types. From these 106,213 gene pairs, we selected 3 gene pairs (Table 2) with the largest geometric mean of the average absolute rank difference in the lymphoma 
Table 1 High-input RNA tissue samples used in this study

\begin{tabular}{lll}
\hline Tissue Sample Type & GEO ID & Sample Size \\
\hline Lymphoma & GSE55267 & 69 \\
Breast cancer & GSE29431 & 54 \\
Lymphoma & GSE53820 & 81 \\
Breast cancer & GSE10780 & 30 \\
COAD & TCGA & 41 \\
Normal tissues paired with COAD & TCGA & 41 \\
Colon tumor tissues & GSE10950 & 25 \\
Colon normal tissues & GSE10950 & 25 \\
Colorectal tumors (CRC) & GSE81861 & 272 \\
Normal mucosas paired with CRC & GSE81861 & 157 \\
\hline
\end{tabular}

tissue samples and the average absolute rank difference in the breast cancer tissues samples (see Materials and Methods). The results showed that when $k=3$ both sensitivity and specificity were $100 \%$. Thus, these three gene pairs with the highest $R_{i j}$ values, as described in Table 2 , were selected as the classification signature. Using the 3 gene pairs as signature, we classified a given sample according to the majority vote rule. If 2 or 3 REOs of the 3 gene pairs in a sample were consistent with the REO patterns in the lymphoma tissue samples, the sample was identified as a lymphoma tissue sample; otherwise, the sample was identified as a breast cancer tissue sample. In the training datasets, obviously, all of the lymphoma tissues samples and the breast cancer tissue samples were correctly classified using the signature. In the independent validation dataset, consisting of 81 high-input RNA samples of lymphoma tissue from the GSE53820 dataset and 30 high-input RNA samples of breast cancer tissue from the GSE10780 dataset, all of the samples were correctly classified.

We further applied the REOs-based signature to distinguish Raji and MCF-7 cell lines profiled with highinput with $100 \mathrm{ng}$ total RNA and low-input samples with as low as $50 \mathrm{pg}$ total RNA from the GSE17565 dataset. All the 8 high-input Raji cell line samples, 8 high-input MCF-7 cell line samples, 12 low-input Raji cell line samples and 12 low-input MCF-7 cell line samples were correctly classified. This case study demonstrates that a REOs-based transcriptional signature identified from the high-input RNA tissue samples can be applied to classify low-input samples robustly.

Table 2 The 3 gene-pair signature

\begin{tabular}{lll}
\hline Gene pair No. & Gene $A^{\text {a }}$ & Gene B \\
\hline 1 & MMP3 & RGS13 \\
2 & EPCAM & CD37 \\
3 & EPCAM & STAP1 \\
\hline
\end{tabular}

${ }^{\mathrm{a}}$ Gene $\mathrm{A}$ had a higher expression level than Gene $\mathrm{B}$ in breast cancer tissues and MCF-7 cell lines
As a second case study, we identified a REO-based signature from high-input RNA samples for discriminating primary colorectal tumors from normal colorectal tissues and showed that it can be robustly applied to low-input RNA samples summarized from single-cell RNA-seq data. Firstly, using the 41 colon adenocarcinoma samples and paired normal samples from TCGA, we identified two lists of gene pairs, each with identical REOs in all samples of the primary colorectal tumor tissue and the corresponding normal tissue, respectively. From the above two lists of gene pairs, 20,390 gene pairs were found to have reversal REOs between the tumor tissues and the normal tissues. Because there were an abundance of dropout events that led to zero expression values for approximately $90 \%$ of the genes measured in the single-cell data, it would be inappropriate to select only a few gene pairs as the diagnostic signature. Therefore, all the reversal gene pairs were directly used as the signature. In the training dataset, the 20,390 gene pairs correctly classified all the cancer and normal samples according to the majority voting rule. Then, we collected an independent dataset from GSE10950 with 25 highinput RNA samples of paired colon tumor tissues and colon normal tissues to validate this signature. Because only 18,227 gene pairs of the 20,390 gene pairs were measured in this dataset by the Illumina human Ref- 8 v2.0 platform, these 18,227 gene pairs were used to classify the samples according to the majority voting rule and all the samples were correctly classified. However, with the same strategy, 272 colorectal tumor epithelial cells and 157 normal epithelial cells from the GSE81861 dataset could not be correctly classified. This result is not surprising since a cell contains only approximately 10 pg RNA and $90 \%$ of genes were measured with zero expression values. The REOs of gene pairs in such small input RNA samples would be unstable as demonstrated above. To address this issue, we constructed a pooled dataset from the single-cell RNA-seq results.

In the GSE81861 dataset, the 272 tumor epithelial cells and the 157 normal epithelial cells were extracted from 11 patients of primary colorectal tumors and paired normal tissues; however, there were no annotation on patients' information. We randomly assigned the 272 colorectal tumor epithelial cells into 11 samples with approximately equal number of cells: 10 samples each with 25 single cells and a sample with 22 single cells. Each simulated disease sample contains approximately $250 \mathrm{pg}$ RNA. Similarly, the 157 normal epithelial cells were also randomly assigned into 10 samples each with 14 single cells and a sample with 17 single cells. Each sample approximately contains $140 \mathrm{pg}$ RNA. In each sample, we calculated the sum of the measurement values for each gene to represent the expression levels of the genes [19]. Then, the REO signature with 20,390 gene pairs 
constructed from the high-input RNA samples was applied to classify the simulated low-input RNA samples from the single-cell data. Because only 18,308 gene pairs of the 20,390 gene pairs were measured in single cells by the Illumina HiSeq 2000 platform, we used the measured 18,308 gene pairs to classify the samples according to the majority voting rule. This random experiment was repeated for 100 times. The results showed that the average sensitivity and specificity were 100 and $73.55 \%$, respectively. As demonstrated in the above Section, the REOs of gene pairs in the input samples with less than 250 pg RNA input tends to be less robust against the amplification bias. Therefore, for the 18,308 gene pairs, we respectively excluded 10 and $20 \%$ of pairs with the smallest average rank differences in either the normal samples or the disease samples, and used the remained gene pairs to classify the samples. For 100 random experiments, while the average sensitivity was kept at $100 \%$, the average specificity increased to $91.82 \%$ (or $100 \%$ ) when $10 \%$ (or $20 \%$ ) of the gene pairs with the smallest average rank differences in either the normal samples or the disease samples were excluded.

\section{Discussion}

It is crucial to develop reliable analysis methods for the precise monitoring of global gene expression levels in limited clinical tissues in many research areas of biological and medical disciplines. For those methods based on quantitative gene expression values such as differential genes and risk score signatures, there exists large uncertainty for the low-input RNA samples due to inherent amplification bias and technical noise in the amplification procedures. However, the relative expression orderings of gene pairs are tolerant to these issues, which suggests us that we should take the advantage of the robustness of REOs to gain more reliable biological insight.

We compared serially diluted RNA samples to evaluate the impact of amplification techniques for low-input RNA samples on the gene expression profile measurements. As displayed in the study, thousands of genes had at least 2 folds-change of expression measurements in the low-input RNA samples compared with the paired high-input RNA samples due to the amplification procedure. Consequently, for the transcriptional signatures based on the quantitative expression levels, the risk threshold values determined from high-input RNA samples could not be applied to low-input RNA samples directly and vice versa. In contrast, we found that approximately $90 \%$ of REOs of gene pairs in high-input RNA samples were maintained in the diluted $1000 \mathrm{pg}$, low-input mRNA samples which were amplified and profiled by Smart-seq, DP-seq and CEL-seq techniques using the Illumina HiSeq 2000 platform. For the lowinput total samples which were amplified and profiled by the Whole-Genome DASL technique using the Illumina HumanRef- 8 v3.0 platform, at least $90 \%$ of REOs of gene pairs in the high-input samples were maintained in the diluted $1000 \mathrm{pg}$ and $250 \mathrm{pg}$ input samples but unstable in the $50 \mathrm{pg}$ and $10 \mathrm{pg}$ input samples to a certain degree.

Our REO-based method facilitates gene expression profiling analysis in the context where the starting RNA material is extremely limited. A problem with the current study is that we cannot find appropriate data to verify the clinical value of the REOs-based signature. For the future study, it is worthwhile to further evaluate the method using clinically meaningful low-input RNA data such like tissues from minimally invasive tissue biopsy techniques and single-cell samples.

\section{Conclusions}

Thousands of genes have at least 2 folds-change of expression measurements in low-input mRNA and total RNA samples compared with the corresponding paired high-input samples. In contrast, most of the REOs of gene pairs in the high-input samples are maintained in the diluted low-input samples. Therefore, REOs-based disease signatures determined from high-input samples can be robustly applied to low-input samples.

\section{Methods}

\section{Data sources and data preprocessing}

All the gene expression data analyzed in this study were downloaded from the GEO database (http://www.ncbi. nlm.nih.gov/geo/), as described in details in Fig. 1 and Table 1. In Fig. 1, there are 2 datasets including mRNA sequencing data and whole genome gene expression data which were used to evaluate the amplification bias. In Table 1, there are, in total, 6 datasets of high-input RNA tissue samples, including 4 sets which were used to obtain the classification signature between breast cancer and lymphoma cancer and 2 datasets which were used to obtain the classification signature between colon tumor tissues and normal tissues.

The gene expression profiles of dataset GSE50856 were measured by the Illumina HiSeq 2000 platform for the low-input mRNA samples collected from day-4 embroid bodies of mouse embryonic stem cells (mESCs) differentiated in serum free media with and without Activin A treatment. The control samples were labeled with "SFM" and the Activin A-treated samples were labeled with "AA1000". The low-input samples were amplified and profiled by Smart-seq, DP-seq and CEL-seq techniques using the Illumina HiSeq 2000 platform, with those of the paired high-input (50 ng) mRNA samples. Based on the amplification methods and cell line treatment status, the dataset was divided into 6 groups: SFM-Smart, SFM-DP, SFM-CEL, AA100-Smart, AA100DP and AA100-CEL. Each group had four input levels, 
$1000 \mathrm{pg}, 100 \mathrm{pg}, 50 \mathrm{pg}$ and $25 \mathrm{pg}$, and each level had two technical replicates. The gene expression profile of dataset GSE17565 was measured by the Illumina HumanRef-8 v3.0 platform for two cell lines, Raji and MCF-7. The dataset was divided into 2 groups: Raji and MCF-7. The low-input total RNA samples were amplified and profiled by the Whole-Genome DASL technique using the Illumina HumanRef- 8 v3.0 platform using the Illumina HumanRef-8 v3.0 platform, with those of the paired high-input (100 ng) total RNA samples. There were four input levels, $1000 \mathrm{pg}, 100,250 \mathrm{pg}$, $50 \mathrm{pg}$ and $2510 \mathrm{pg}$ as well for both cell lines, and every input level had two technical replicates (Fig. 1).

For the GSE50856 dataset, we downloaded the mappable reads that fell onto gene's exons. The experiments of standard RNA-seq, Smart-seq and DP-seq are single-end RNAseq where every read corresponds to a single fragment. Thus, the RPKM (reads per kilobase of exon model per million mapped reads) and FPKM (fragments per kilobase of exon model per million mapped reads) metrics are conceptually analogous [35, 36], which could be used to quantify the gene expression level. The RPKM metric was estimated by the formula [37]: $\mathrm{R}=\left(10^{\wedge} 9^{*} \mathrm{C}\right) / \mathrm{NL}$, where $\mathrm{C}$ is the number of mapped reads that fell onto the gene's exons, $\mathrm{N}$ is the total number of mapped reads in the experiment, and $\mathrm{L}$ is the sum of the exons in base pairs. On the other hand, The experiment of CEL-seq is paired-end sequencing where two reads correspond to a single fragment and only FPKM could be used to quantify the gene expression level. For the paired-end experiment, the FPKM value would be half of the RPKM value. This is not always true because in some cases only one of the two reads belonging to a fragment might be mapped. However, for most applications this simplification works [35]. The mouse mm9 genome was used for the genome annotation. By transforming the gene bank accession ID providing in the GSE50856 dataset into Entrez gene ID through the Source Batch Search database (http:// source-search.princeton.edu/cgi-bin/source/sourceBatch-

Search), 20,541 genes were analyzed in this dataset.

For dataset GSE17565 measured by Illumina HumanRef8 v3.0 platform, dataset GSE10950 measured by Illumina humanRef-8 v2.0 platform and dataset GSE81861 measured by Illumina HiSeq 2000 platform, we directly downloaded the processed data. For 4 datasets of the expression profiles measured by Affymatrix microarrays, the raw expression data (.CEL files) were preprocessed using the Robust Multiarray Average algorithm [38]. For the data from TCGA, the level 3 RNA-seq datasets (RNAseqV2 RSEM) of mRNA were downloaded from the Broad Institute, Firehose (http://gdac.broadinstitute.org/runs/stddata_2016_01_28/).

\section{Evaluation on amplification bias by fold change}

For each of the measured genes, we calculated the average of its expression values in the technical replicates for the low input and the paired high input RNA samples, respectively, and then calculated the fold changes (FCs) between the low input RNA samples and the paired high input RNA samples. We also calculated the FC between every paired low input RNA technical replicate and high input RNA technical replicates, and then calculated the coefficient of variation (CV) of the FCs.

\section{Evaluation on REOs of gene pairs}

Highly stable REOs of the gene pairs were obtained respectively from high-input RNA samples and low input RNA samples. We defined a REO as highly stable if the gene pair had identical REO direction in both technical replicates of one sample. The details are as following. The comparison of two genes in a gene pair $\left(G_{i}, G_{j}\right)$ was viewed as an event with only two possible outcomes: the expression level of $G_{i}$ was either higher or lower than that of $G_{j}$ and the relative expression ordering was denoted as $G_{i}>G_{j}$ or $G_{i}<G_{j}$. If the REO of a pair was maintained in more than $99 \%$ of samples, the pair was called a highly stable gene pair. The REOs of two genes with small rank difference (i.e., close expression levels) tend to be unstable due to measurement variations.

To compare two lists of stable REOs, the consistency score, which was defined as $k / n$, was calculated, where $n$ was the number of the gene pairs in the high-input RNA samples and $k$ was the number of gene pairs with the consistent REOs in both the high-input RNA samples and low-input RNA samples.

\section{REOs-based signature from high-input RNA samples for discriminating cancer tissues}

First, we identified gene pairs each with identical REO in all samples of the two types of tissues, respectively, but with reversal REO patterns between the two types of samples. Then, we calculated the reversal degree for each gene pair as following equation,

$$
\bar{R}_{i j}=\sqrt{\bar{R}_{i j(\mathrm{lym})} \bar{R}_{i j(\mathrm{bre})}}
$$

where $R_{i j(\mathrm{lym})}$ and $R_{\mathrm{ij}(\mathrm{bre})}$ are the arithmetic means of the absolute rank differences of the gene pair $(i, j)$ in all samples of the two types of tissues, respectively.

Second, the gene pairs with reversal REOs were sorted in a descending order according to their reversal degrees. Obviously, the larger the $R_{i j}$ value, the larger the reversal degree of the REO is between the two types of samples. Third, we selected the top $k$ gene pairs, where $k$ is an odd integer ranging from 1 to the total number of candidate gene pairs to classify the samples based on the majority vote rule. The value of $k$ was chosen as the smallest number of gene pairs that reached the highest geometric mean of the sensitivity and specificity in the classification tests. Then, the selected gene-pair signature 
was tested in independent tissue samples measured with high-input RNA and in the cell line data measured with low-input RNA.

\section{Performance evaluation}

We called lymphoma tissue samples, colorectal tumor epithelial cells as positive samples, breast cancer tissue samples, Normal mucosa's epithelial cells paired with colorectal tumor as negative samples, and evaluated the performance of the classification signature using sensitivity and specificity which are calculated as follows:

$$
\begin{aligned}
& \text { Sensivity }=\frac{T P}{T P+F N} \\
& \text { Specificity }=\frac{T N}{T N+F P}
\end{aligned}
$$

where TP, TN, FP and FN denote the number of true positives, true negatives, false positives and false negatives, respectively.

\section{Statistical software for analysis}

All statistical analyses were performed using the R 3.1.3 (http://www.r-project.org/). The main analyses codes are provided in the (Additional file 2).

\section{Additional files}

Additional file 1: Figure S1. The coefficient of variation (CV) of FCs. (a) The coefficient of variation (CV) of FCs in the three groups of SFM-DP, SFM-CEL and SFM-Smart respectively in the $25 \mathrm{pg}, 50 \mathrm{pg}, 100 \mathrm{pg}$ and $1000 \mathrm{pg}$ RNA quantity (b) (c) Similar as the Figure a. Figure S2. Maintenance of REOs after excluding 0 to $10 \%$ gene pairs. (a) The consistency scores between high-input RNA samples and low-input RNA samples of all gene pairs (blue) and after excluding $10 \%$ of the pairs with the smallest expression differences in the paired high-input RNA samples (pink) in the group of AA100-Smart (b) (c) (d) Similar as the Figure a. Figure S3. Maintenance of REOs after excluding 0 to $30 \%$ gene pairs. The consistency scores between high-input RNA samples and low-input RNA samples of all gene pairs, after excluding 0, 5, 10, 15, 20 and $30 \%$ of the pairs with the smallest expression differences in the paired high-input RNA samples in the group of AA100-Smart (b) (c) (d) Similar as the Figure a. (PDF 606 kb)

Additional file 2: The main analyses codes used in this research. (R 4 kb)

\section{Abbreviations}

AA100: Activin A treatment; AA100-CEL: Mouse embryonic stem cells differentiated in control serum free media (SFM) and the collected RNA amplified and profiled by CEL-seq using the Illumina HiSeq 2000 platform; AA100-DP: Mouse embryonic stem cells differentiated in control serum free media (SFM) and the collected RNA amplified and profiled by DP-seq using the Illumina HiSeq 2000 platform; AA100-Smart: Mouse embryonic stem cells subjected to Activin A treatment (AA100) and the collected RNA amplified and profiled by Smart-seq using the Illumina HiSeq 2000 platform; CELseq: Cell expression by linear amplification and sequencing; COAD: Colon adenocarcinoma; CRC: Colorectal tumors; DP-seq: Designed Primer-based RNA-sequencing strategy; FC: Fold change; GEO: Gene Expression Omnibus; REOs: Within-sample relative expression orderings; SFM: Serum free media; SFM-CEL: Mouse embryonic stem cells differentiated in control serum free media (SFM) and the collected RNA amplified and profiled by CEL-seq using the Illumina HiSeq 2000 platform; SFM-DP: Mouse embryonic stem cells differentiated in control serum free media (SFM) and the collected RNA amplified and profiled by DP-seq using the Illumina HiSeq 2000 platform;
SFM-Smart: Mouse embryonic stem cells differentiated in control serum free media (SFM) and the collected RNA were amplified and profiled by Smartseq using the Illumina HiSeq 2000 platform; SMART: Switching mechanism at 5 '-end of the RNA transcript

\section{Acknowledgements}

Thank to all the individuals who participated in this study. We would also like to acknowledge the resources at GEO that facilitated this research.

\section{Funding}

This work was supported by National Natural Science Foundation of China (Grant numbers: 81,372,213, 81,572,935 and 21,534,008) and the Joint Technology Innovation Fund of Fujian Province (Grant number: 2016Y9044).

Availability of data and materials

Previously data analyzed in this study should be requested from the authors of the original publications. Please see methods cohort description (Table 1), for references to these publications.

\section{Authors' contributions}

ZG, XLW and HPL conceived the project. HPL, YWL, JH and QZG performed computational experiments. HPL, HDY, RC and WCZ designed data analyses. $\mathrm{HPL}, \mathrm{KS}, \mathrm{HC}$ and $\mathrm{YG}$ interpreted data. HPL, XLW and ZG wrote the manuscript. All authors contributed to the preparation of the manuscript. All authors read and approved the final manuscript.

Ethics approval and consent to participate

Not applicable.

\section{Consent for publication}

Not applicable.

\section{Competing interests}

The authors declare that they have no competing interests.

\section{Publisher's Note}

Springer Nature remains neutral with regard to jurisdictional claims in published maps and institutional affiliations.

\section{Author details}

'Department of Bioinformatics, Key Laboratory of Ministry of Education for Gastrointestinal Cancer, School of Basic Medical Sciences, Fujian Medical University, Fuzhou 350122, China. ${ }^{2}$ Fujian Key Laboratory of Tumor Microbiology, Fujian Medical University, Fuzhou 350122, China. ${ }^{3}$ Department of Systems Biology, College of Bioinformatics Science and Technology, Harbin Medical University, Harbin 150086, China. ${ }^{4}$ Key Laboratory of Medical bioinformatics, Fujian Province, China.

Received: 2 June 2017 Accepted: 3 November 2017

Published online: 28 November 2017

\section{References}

1. De Rienzo A, Yeap BY, Cibas ES, Richards WG, Dong L, Gill RR, Sugarbaker DJ, Bueno R. Gene expression ratio test distinguishes normal lung from lung tumors in solid tissue and FNA biopsies. J Mol Diagn. 2014;16(2):267-72.

2. Libby DM, Smith JP, Altorki NK, Pasmantier MW, Yankelevitz D, Henschke Cl. Managing the small pulmonary nodule discovered by CT. Chest. 2004; 125(4):1522-9.

3. Knudsen BS, Kim HL, Erho N, Shin H, Alshalalfa M, Lam LL, Tenggara I, Chadwich K, Van Der Kwast T, Fleshner N, et al. Application of a clinical wholeTranscriptome assay for staging and prognosis of prostate cancer diagnosed in needle Core biopsy specimens. J Mol Diagn. 2016;18(3):395-406.

4. Cabanski CR, Magrini V, Griffith M, Griffith OL, McGrath S, Zhang J, Walker J, Ly A, Demeter R, Fulton RS, et al. cDNA hybrid capture improves transcriptome analysis on low-input and archived samples. J Mol Diagn. 2014;16(4):440-51

5. Soeda H, Sakudo F. NaCl and water responses across the frog tongue epithelium in vitro. Fukuoka Shika Daigaku Gakkai zasshi. 1990;17(3):251-9.

6. Wen L, Tang F. Single-cell sequencing in stem cell biology. Genome Biol. 2016;17:71. 
7. Liang J, Cai W, Sun Z. Single-cell sequencing technologies: current and future. J Genet Genomics. 2014;41(10):513-28.

8. Datta S, Malhotra L, Dickerson R, Chaffee S, Sen CK, Roy S. Laser capture microdissection: big data from small samples. Histol Histopathol. 2015; 30(11):1255-69.

9. Ramskold D, Luo S, Wang YC, Li R, Deng Q, Faridani OR, Daniels GA, Khrebtukova I, Loring JF, Laurent LC, et al. Full-length mRNA-Seq from single-cell levels of RNA and individual circulating tumor cells. Nat Biotechnol. 2012;30(8):777-82.

10. Bhargava V, Ko P, Willems E, Mercola M, Subramaniam S. Quantitative transcriptomics using designed primer-based amplification. Sci Rep. 2013;3:1740

11. Hashimshony T, Wagner F, Sher N, Yanai I. CEL-Seq: single-cell RNA-Seq by multiplexed linear amplification. Cell Rep. 2012;2(3):666-73.

12. Bhargava V, Head SR, Ordoukhanian P, Mercola M, Subramaniam S. Technical variations in low-input RNA-seq methodologies. Sci Rep. 2014;4:3678.

13. Kolodziejczyk AA, Kim JK, Svensson V, Marioni JC, Teichmann SA. The technology and biology of single-cell RNA sequencing. Mol Cell. 2015;58(4): 610-20

14. Boelens MC, te Meerman GJ, Gibcus JH, Blokzijl T, Boezen HM, Timens W, Postma DS, Groen HJ, van den Berg A. Microarray amplification bias: loss of $30 \%$ differentially expressed genes due to long probe - poly(a)-tail distances. BMC Genomics. 2007;8:277.

15. Spiess AN, Mueller N, Ivell R. Amplified RNA degradation in T7-amplification methods results in biased microarray hybridizations. BMC Genomics. 2003;4(1):44.

16. Oshlack A, Wakefield MJ. Transcript length bias in RNA-seq data confounds systems biology. Biol Direct. 2009;4:14

17. van Haaften RI, Schroen B, Janssen BJ, van Erk A, Debets JJ, Smeets HJ, Smits JF, van den Wijngaard A, Pinto YM, Evelo CT. Biologically relevant effects of mRNA amplification on gene expression profiles. BMC Bioinformatics. 2006;7:200.

18. Degrelle SA, Hennequet-Antier C, Chiapello H, Piot-Kaminski K, Piumi F, Robin S, Renard JP, Hue I. Amplification biases: possible differences among deviating gene expressions. BMC Genomics. 2008;9:46.

19. Tariq MA, Kim HJ, Jejelowo O, Pourmand N. Whole-transcriptome RNAseq analysis from minute amount of total RNA. Nucleic Acids Res. 2011;39(18):e120.

20. Faherty SL, Campbell CR, Larsen PA, Yoder AD. Evaluating whole transcriptome amplification for gene profiling experiments using RNA-Seq. BMC Biotechnol. 2015;15:65.

21. Clement-Ziza M, Gentien D, Lyonnet S, Thiery JP, Besmond C, Decraene C. Evaluation of methods for amplification of picogram amounts of total RNA for whole genome expression profiling. BMC Genomics. 2009;10:246.

22. Qi L, Chen L, Li Y, Qin Y, Pan R, Zhao W, Gu Y, Wang H, Wang R, Chen X, et al. Critical limitations of prognostic signatures based on risk scores summarized from gene expression levels: a case study for resected stage I non-small-cell lung cancer. Brief Bioinform. 2016:17(2):233-42.

23. Xu H, Guo X, Sun Q, Zhang M, Qi L, Li Y, Chen L, Gu Y, Guo Z, Zhao W. The influence of cancer tissue sampling on the identification of cancer characteristics. Sci Rep. 2015;5:15474.

24. Cheng J, Guo Y, Gao Q, Li H, Yan H, Li M, Cai H, Zheng W, Li X, Jiang W, et al. Circumvent the uncertainty in the applications of transcriptional signatures to tumor tissues sampled from different tumor sites. Oncotarget. 2017;8(18):30265-75

25. Freidin MB, Bhudia N, Lim E, Nicholson AG, Cookson WO, Moffatt MF. Impact of collection and storage of lung tumor tissue on whole genome expression profiling. J Mol Diagn. 2012;14(2):140-8.

26. Chen R, Guan Q, Cheng J, He J, Liu H, Cai H, Hong G, Zhang J, Li N, Ao L, et al. Robust transcriptional tumor signatures applicable to both formalinfixed paraffin-embedded and fresh-frozen samples. Oncotarget. 2017;8(4): 6652-62.

27. Tan AC, Naiman DQ, Xu L, Winslow RL, Geman D. Simple decision rules for classifying human cancers from gene expression profiles. Bioinformatics. 2005;21(20):3896-904

28. Geman D, d'Avignon C, Naiman DQ, Winslow RL. Classifying gene expression profiles from pairwise mRNA comparisons. Stat Appl Genet Mol Biol. 2004;3:Article19.

29. Zhao W, Chen B, Guo X, Wang R, Chang Z, Dong Y, Song K, Wang W, Qi L, $\mathrm{Gu} Y$, et al. A rank-based transcriptional signature for predicting relapse risk of stage II colorectal cancer identified with proper data sources. Oncotarget. 2016;7(14):19060-71.

30. Qi L, Li Y, Qin Y, Shi G, Li T, Wang J, Chen L, Gu Y, Zhao W, Guo Z. An individualised signature for predicting response with concordant survival benefit for lung adenocarcinoma patients receiving platinum-based chemotherapy. Br J Cancer. 2016:115(12):1513-9.

31. Cai H, Li X, Li J, Ao L, Yan H, Tong M, Guan Q, Li M, Guo Z. Tamoxifen therapy benefit predictive signature coupled with prognostic signature of post-operative recurrent risk for early stage ER+ breast cancer. Oncotarget. 2015;6(42):44593-608

32. Li X, Cai H, Zheng W, Tong M, Li H, Ao L, Li J, Hong G, Li M, Guan Q, et al. An individualized prognostic signature for gastric cancer patients treated with 5-fluorouracil-based chemotherapy and distinct multi-omics characteristics of prognostic groups. Oncotarget. 2016;7(8):8743-55.

33. Ao L, Song X, Li X, Tong M, Guo Y, Li J, Li H, Cai H, Li M, Guan O, et al. An individualized prognostic signature and multiomics distinction for early stage hepatocellular carcinoma patients with surgical resection. Oncotarget. 2016;7(17):24097-110.

34. Guan Q, Chen R, Yan H, Cai H, Guo Y, Li M, Li X, Tong M, Ao L, Li H, et al. Differential expression analysis for individual cancer samples based on robust within-sample relative gene expression orderings across multiple profiling platforms. Oncotarget. 2016;7(42):68909-20.

35. Vikman P, Fadista J, Oskolkov N. RNA sequencing: current and prospective uses in metabolic research. J Mol Endocrinol. 2014;53(2):R93-101.

36. Trapnell C, Williams BA, Pertea G, Mortazavi A, Kwan G, van Baren MJ, Salzberg SL, Wold BJ, Pachter L. Transcript assembly and quantification by RNA-Seq reveals unannotated transcripts and isoform switching during cell differentiation. Nat Biotechnol. 2010;28(5):511-5.

37. Mortazavi A, Williams BA, McCue K, Schaeffer L, Wold B. Mapping and quantifying mammalian transcriptomes by RNA-Seq. Nat Methods. 2008:5(7):621-8.

38. Irizarry RA, Hobbs B, Collin F, Beazer-Barclay YD, Antonellis KJ, Scherf U, Speed TP. Exploration, normalization, and summaries of high density oligonucleotide array probe level data. Biostatistics. 2003;4(2):249-64.

\section{Submit your next manuscript to BioMed Central and we will help you at every step:}

- We accept pre-submission inquiries

- Our selector tool helps you to find the most relevant journal

- We provide round the clock customer support

- Convenient online submission

- Thorough peer review

- Inclusion in PubMed and all major indexing services

- Maximum visibility for your research

Submit your manuscript at www.biomedcentral.com/submit
) Biomed Central 